\title{
Multisite two-photon imaging of neurons on multielectrode arrays
}

Steve M. Potter, Natalia Lukina, Kenneth J. Longmuir, Yan Wu

Steve M. Potter, Natalia Lukina, Kenneth J. Longmuir, Yan Wu, "Multisite two-photon imaging of neurons on multielectrode arrays," Proc. SPIE 4262, Multiphoton Microscopy in the Biomedical Sciences, (24 April 2001); doi: $10.1117 / 12.424542$

SPIE Event: BiOS 2001 The International Symposium on Biomedical Optics, 2001, San Jose, CA, United States 


\title{
Multi-site two-photon imaging of neurons on multi-electrode arrays
}

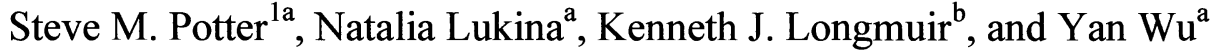 \\ ${ }^{a}$ California Institute of Technology, 156-29 Div. of Biology, Pasadena, CA 91125 \\ ${ }^{b}$ Dept. of Physiology and Biophysics, College of Medicine, University of California, Irvine, CA 92697
}

\begin{abstract}
We wish to understand how neural systems store, recall, and process information. We are using cultured networks of cortical neurons grown on microelectrode arrays as a model system for studying the emergent properties of ensembles of living neurons. We have developed a 2-way communication interface between the cultured network and a computer-generated animal, the Neurally Controlled Animat. Neural activity is used to control the behavior of the Animat, and 2-photon timelapse imaging is carried out in order to observe the morphological changes that might underlie changes in neural processing. The 2-photon microscope is ideal for repeated imaging over hours or days, with sub-micron resolution and little photodamage. We have designed a computer-controlled microscope stage that allows imaging several locations in sequence, in order to collect more image data. For the latest progress, see: http://www.caltech.edu/ pinelab/PotterGroup.htm
\end{abstract}

Keywords: Two-photon laser scanning microscopy, multiphoton, multielectrode array, microelectrode array, green fluorescent protein, liposomes, animat, cortex cell culture

\section{INTRODUCTION}

Thanks to the detailed neuron drawings made by Santiago Ramon y Cajal a century ago, and the work of many other neuroanatomists since, it is well established that brain cells come in a wider variety of shapes and sizes than do those of any other tissue. This complexity of morphology and interconnectivity allows a level of information processing and storage several orders of magnitude greater that the best supercomputers built today. There is evidence that as we learn, the shape of our neurons changes. Most of this evidence is obtained by comparing the brains of animals raised in enriched vs. boring environments, ${ }^{1}$ after they have been sacrificed. Much has been gleaned from this approach, but it is difficult to relate the post-mortem morphology to the electrical activity that was present in the neural tissue while it was alive and learning, and controlling the animal's behavior.

We are developing a number of tools aimed at studying the morphological dynamics of a living neuronal network during the learning process, while simultaneously monitoring its electrical activity. We grow dissociated brain cell cultures (including neurons and glia) on glass dishes with arrays of embedded electrodes (Fig. 1) (multi-electrode arrays, MEAs). ${ }^{2,3}$ These electrodes are capable of recording and stimulating the neurons extracellularly and non-destructively. We have developed new techniques for maintaining primary neural cultures for months. ${ }^{4}$ Combining MEA technology with 2-photon time-lapse microscopy opens up new lines of inquiry not possible in living animals: we can follow the electrical activity and morphological changes in hundreds of neurons for weeks or months. Compared to in vivo studies, cultured networks offer unparalleled access for long-term electrical and pharmacological studies; every neuron in the network can be imaged at the submicron level for hours or days. We hope this will enable us to determine the causal interrelationships between neural morphology and network activity.

\footnotetext{
${ }^{1}$ Corresponding author, spotter@gg.caltech.edu, http://www.caltech.edu/ pinelab/steve.html
} 

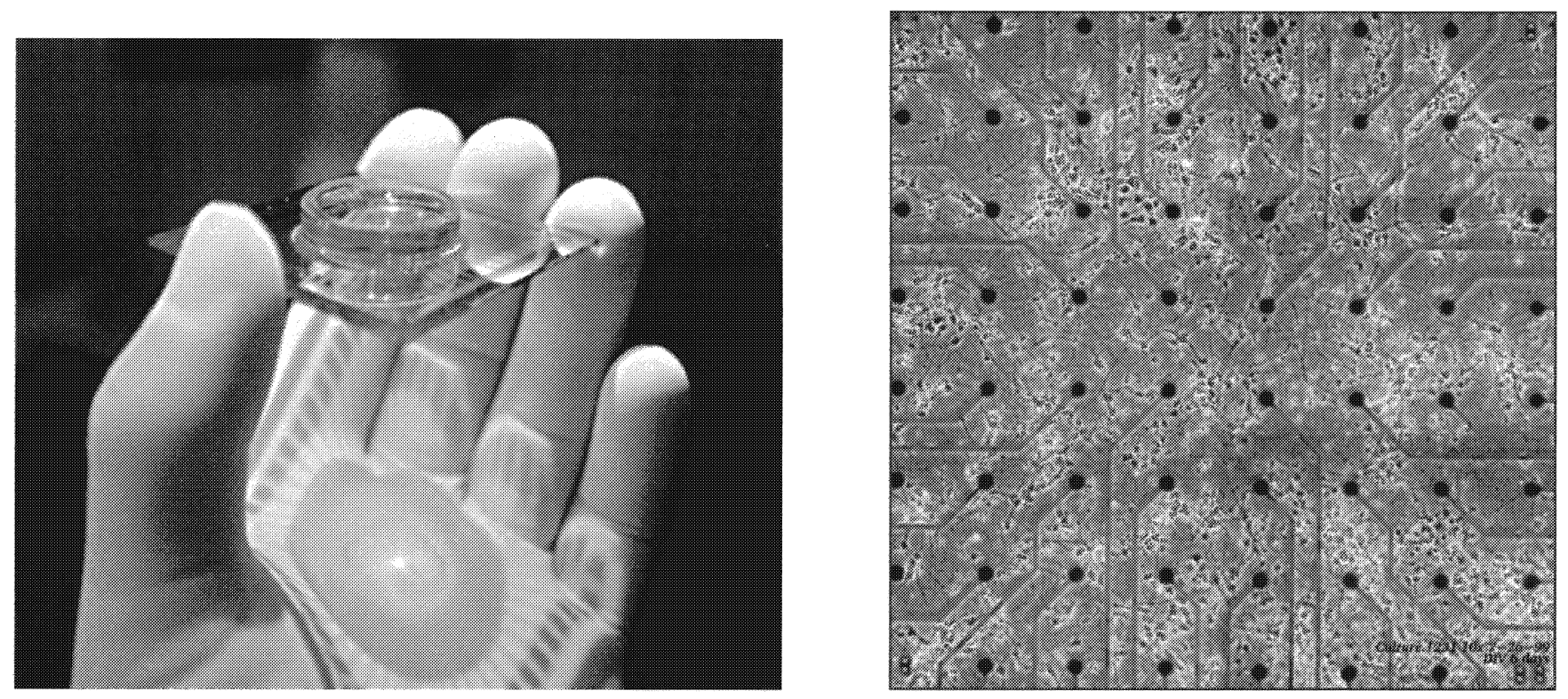

Fig. 1. 60-electrode multi-electrode array culture dish, made by Multichannel Systems. A six-day old culture of cortical cells (right) has already begun to form a synaptically interconnected network, producing coordinated spontaneous electrical activity. Electrodes (dark circles) are $10 \mu \mathrm{m}$ diameter, and $100 \mu \mathrm{m}$ apart. Cultures are prepared from embryonic day 18 rat cortex by enzymatic dissociation ${ }^{5}$ and grown in standard serum-containing medium. ${ }^{6}$

\section{THE NEURALLY-CONTROLLED ANIMAT FOR IN VITRO LEARNING STUDIES}

Rat cortical networks begin to grow axons and dendrites within hours of plating (Fig. 5), and by one week in vitro, have formed numerous synaptic connections. We and others ${ }^{7,9}$ have observed that after two weeks in vitro, the network exhibits rhythmic patterns of coordinated activity bursts with a period of several seconds, as recorded via the MEA electrodes. After one month, the bursting is less periodic and less synchronous across the network, exhibiting a complexity that may represent a greater capacity for information storage and processing. ${ }^{10}$ These patterns can be influenced by extracellular electrical stimulation, suggesting that the strengths of the synapses have changed, altering the network dynamics. ${ }^{11}$

How can we relate this to learning in animals? What does it mean to study learning in vitro? "Learning" usually has a behavioral definition, such as "a lasting change in behavior resulting from experience." An isolated culture of brain cells cannot behave, and therefore one can only speak of studying synaptic plasticity, and causing a change in the response of the cells. To behave, the system needs a body with effectors. Therefore, we created a simulated creature on the computer whose behavior is controlled by the distributed activity in the MEA culture. "Animats" are simulated animals, either on a computer or in robot form, ${ }^{12}$ and this is the first neurally-controlled animat. ${ }^{13}$ As the animat interacts with its simulated environment, it receives sensory and kinesthetic input. These are fed back to the culture, in real-time, as spatio-temporal patterns of electrical pulses on the substrate electrodes (Fig. 2). We have recently closed the loop, so that activity in the cultured net can trigger its own stimulation in less than $100 \mathrm{msec}$, and we are now carrying out experiments designed to detect long-term (hours or days) changes in network activity that we map onto learning in the Animat. The mappings we have chosen so far are arbitrary and simplistic, but in the future we anticipate that a number of learning paradigms traditionally carried out on animals, such as classical conditioning, can also be conducted using a neurally controlled animat. 


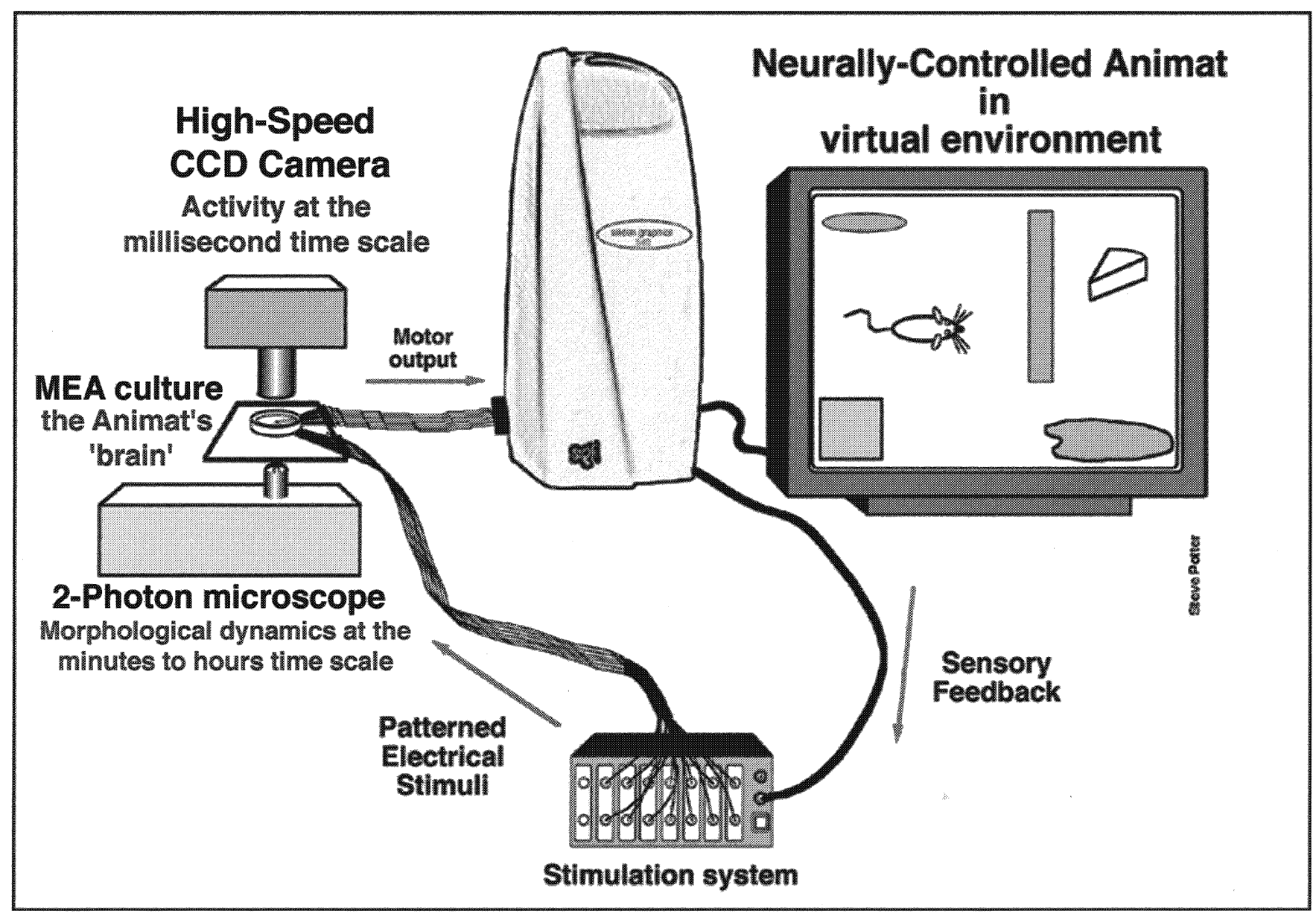

Fig. 2 The Neurally-Controlled Animat, a new tool for studying learning in vitro. A neuronal network is cultured on a multielectrode array. Signals produced by the ongoing activity in the network are collected and processed in real-time by a Silicon Graphics 540 quad-processor computer, to control the behavior of a simulated animal (the animat). As the animat interacts with its simulated environment, sensory input, as well as its own proprioceptive input, is fed back to the culture by a multichannel stimulation system, as spatio-temporal patterns of electrical pulses. Recurring activity patterns are arbitrarily assigned to certain behaviors; if those patterns change as a result of the stimulation feedback, then this can be considered learning. Because the network is growing in a monolayer on a glass substrate, we can image its electrical dynamics on short time scales using voltage-sensitive dyes and a high-speed movie camera, ${ }^{14}$ and we can image morphological dynamics on long time scales using 2-photon time-lapse microscopy.

\section{TWO-PHOTON MICROSCOPY}

Two-photon laser-scanning microscopy ${ }^{15}$ has made it possible to conduct repeated $4 \mathrm{D}(3 \mathrm{D}+$ time $)$ imaging of living specimens with submicron resolution. ${ }^{16,17}$ By using pulsed infrared (IR) light, fluorescence excitation is localized to focus of the laser beam, which is raster-scanned across the specimen one focal plane at a time. Only at the focus is there a reasonable probability that two IR photons will hit a fluorescent molecule at the same time, and excite it as if a single visible photon of twice the energy had been absorbed. Thus phototoxicity and photobleaching are dramatically reduced, compared to fluorescence imaging using visible light, where excitation happens above and below the focal plane as well. The height of the point-spread function of 2-photon excitation is on the order of one micron, with the $40 \mathrm{x} / 0.75$ numerical aperture waterimmersion objective used here. Because cultured cells are 10-15 $\mu \mathrm{m}$ thick, the reduction in photodamage afforded by 2photon excitation is even useful for imaging planar cultures with 2D scans, such as cortical cultures growing on MEAs. 


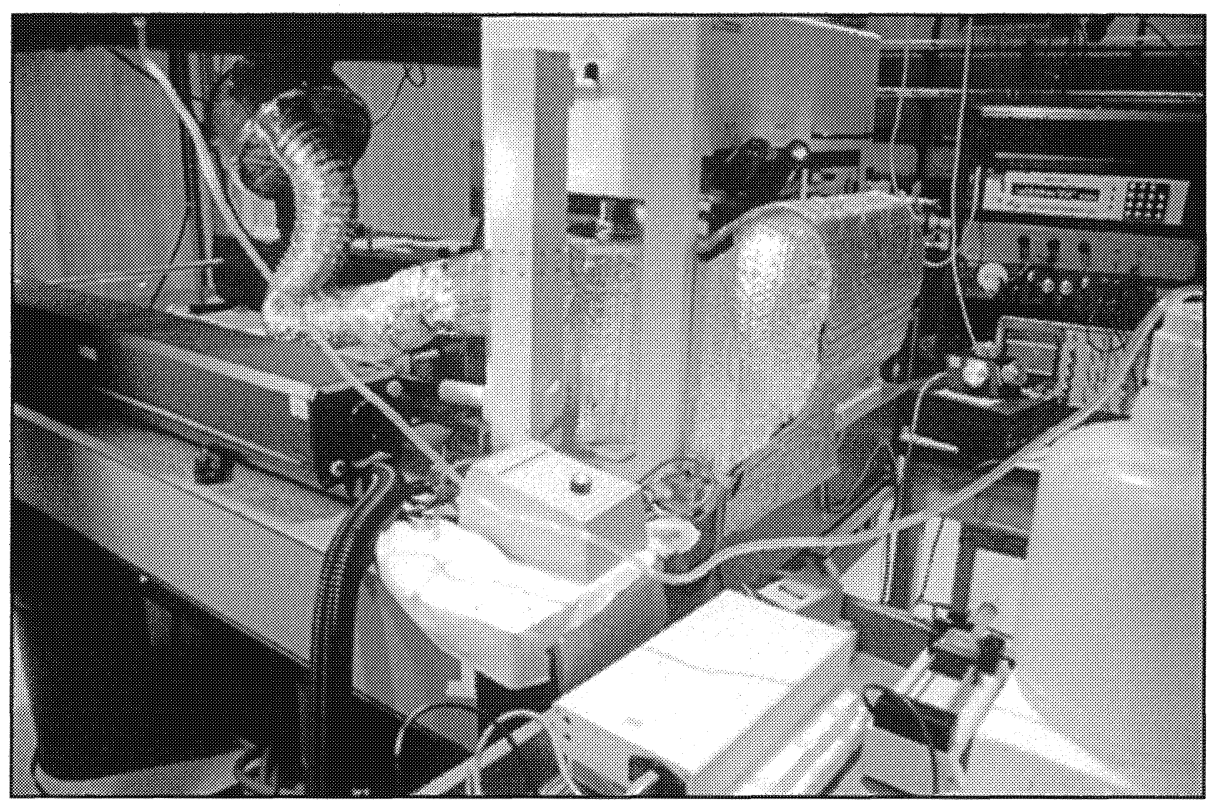

Fig. 3. 2-photon laser-scanning microscope, a converted Molecular Dynamics Sarastro 2000 confocal. In the background, left, are the pump laser and pulsed-IR Ti:Sapphire laser (Coherent Mira900). We now use a smaller, all sold-state pump laser, the Coherent Verdi (not shown). The microscope is enclosed in an opaque insulating chamber and heated to rat body temperature using a chicken egg incubator connected by flexible ducting. In the foreground is a "heartlung' machine that oxygenates and pumps warmed medium to the cultured net.

Our 2-photon microscope (Fig. 3) consists of a converted Molecular Dynamics Sarastro 2000 confocal with an upright Nikon Optiphot2 microscope. Details of the conversion can be found elsewhere. ${ }^{18,19} \mathrm{We}$ use a Coherent Verdi $5 \mathrm{~W}$ pump laser and Coherent Mira900 Ti:Sapphire pulsed IR laser. For these experiments, the laser was tuned to $875 \mathrm{~nm}$, and attenuated to $30 \%$. The microscope is enclosed in Mylar bubble-plastic insulation, and this enclosure is connected to a chicken egg incubator (Lyon Electric Co.) that gently warms the specimen and optics to $37^{\circ} \mathrm{C}$. This opaque enclosure allows imaging at physiologically relevant temperatures, reduces background signal from room lighting, and eliminates drift in focus due to temperature fluctuations in the room.

The field of view of our 40x objective is $256 \mu \mathrm{m}$ across at minimum zoom, and $64 \mu \mathrm{m}$ across when fully zoomed-in $(0.125$ $\mu \mathrm{m}$ pixels, $512 \times 512$ ). Our MEAs consist of $8 \times 8$ electrode grids (minus the corner electrodes) with 100 or $200 \mu \mathrm{m}$ spacing, so the entire array of 60 electrodes is either $700 \mu \mathrm{m}$ or $1.4 \mathrm{~mm}$ across. Thus, any given image is but a small subset of the entire network over the electrodes. To allow us to image more of the network than one field of view per imaging session, we rigged up a Sutter MP285 motorized micromanipulator to move the specimen from one location to another. The 'RoboStage' (Fig. 4) is controlled through the serial port of the same computer that collects the image data (SGI Indigo). A custom Unix program was written to interface the MP285 control box with the ImageSpace scanning software. This program allows the user to select several adjacent or distant sites on the MEA to image. The RoboStage then moves the specimen to each site in turn, repeatedly, to allow the collection of several time-lapse movies in one imaging session. The MP285 has a $2.5 \mathrm{~cm}$ travel in $\mathrm{X}, \mathrm{Y}$, and $\mathrm{Z}$, with a reproducibility finer than one micron.

A pair of sequentially-imaged sites is shown in Fig. 5, along with a minimum zoom time-lapse sequence of the 2-photon fluorescence of neurons establishing connections soon after plating. In order to visualize the processes of the neurons more clearly in our unusually dense cultures $(\sim 10,000$ cells per sq. $\mathrm{mm})$, cells were labeled with the lipophilic dye, $\mathrm{DiO}^{20}$, and mixed with an excess of unlabeled cells, in suspension before plating. This allows us to observe morphological dynamics for up to two weeks after staining. In cases where we wish to follow morphological dynamics in more mature cultures, cells are labeled by exposing them to liposomes containing the gene for the enhanced green fluorescent protein (eGFP, Gene Therapy Systems, San Diego), along with a fusogenic peptide, a component to prevent serum inactiviation, and a cationic nuclear localization signal. ${ }^{21}$ This novel gene delivery vector results in bright GFP labeling within one day in $1-5 \%$ of the cells in the culture. The dendrites of 2-week-old cultured neurons have many spines, and our two-photon time-lapse imaging shows that the morphology of these post-synaptic structures can be quite dynamic (Fig. 6). It is worth noting that some spines are much more dynamic than are others on the same dendrite, which suggests that the ongoing spontaneous electrical activity in the culture is inducing synapse-specific remodeling. 


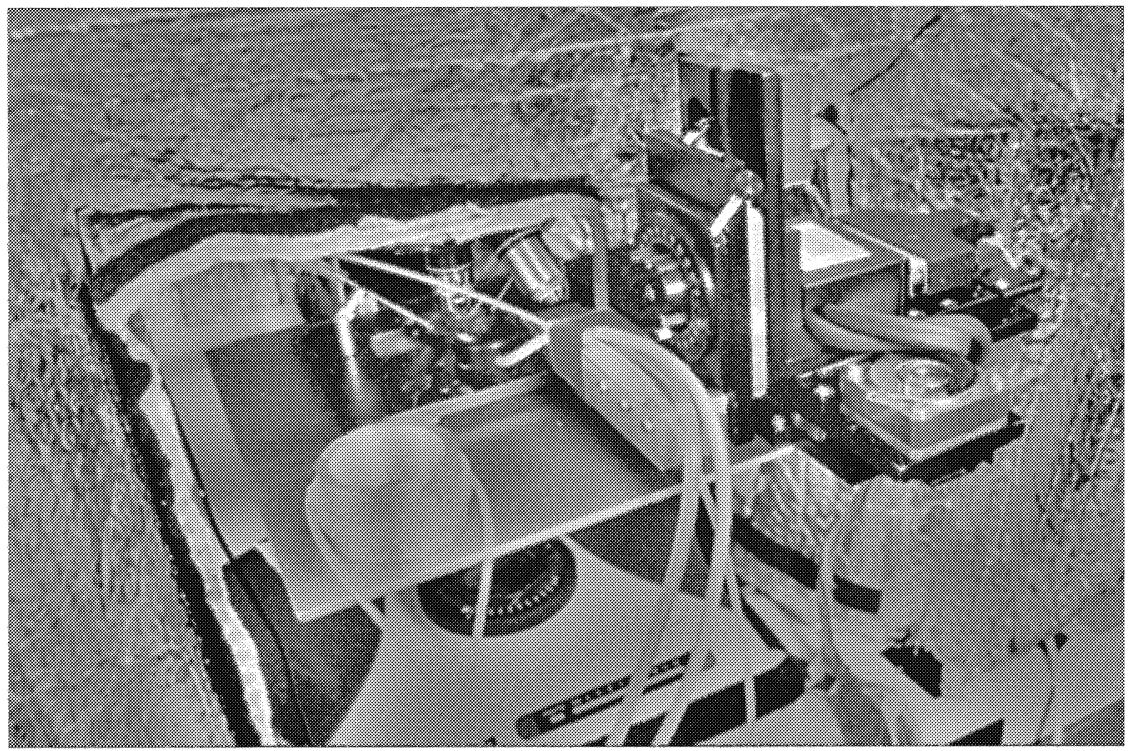

Fig. 4. The RoboStage, consisting of a Sutter MP-285 micromanipulator, is connected via the serial port to the computer that controls the 2-photon microscope. This view inside the environmental enclosure shows the culture dish holder bolted to the manipulator, which is in turn bolted to the stage of the microscope. The manipulator is programmed to move the specimen to several fields of view, repeatedly, in sequence, under the stationary objective lens. A perfusion system keeps the medium in the culture fresh and allows pharmacological manipulation of the network during time-lapse imaging.
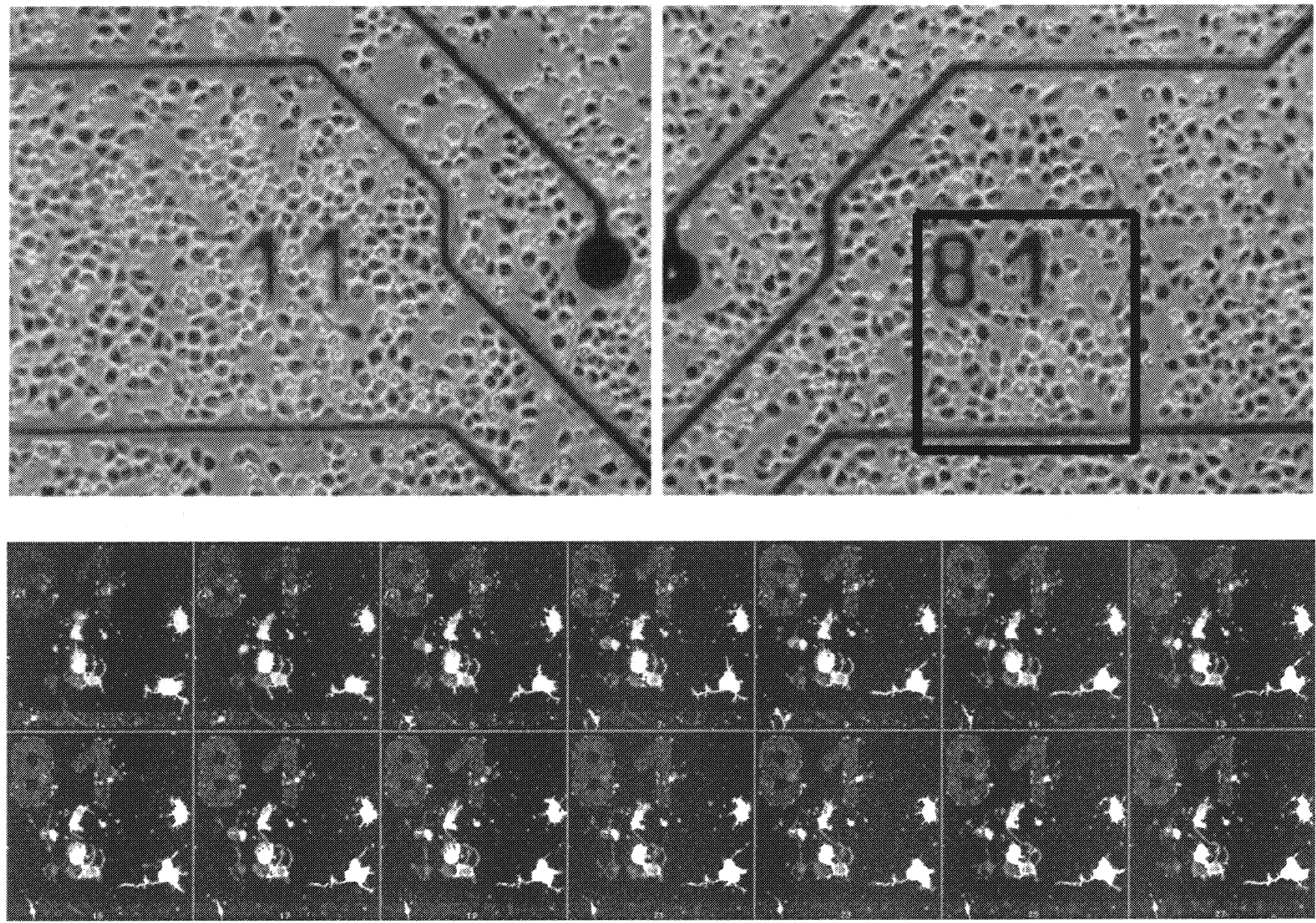

Fig.5. Rat cortical cell culture on a multi-electrode array. Cells labeled in suspension with DiO were mixed with an excess of unlabeled cells and plated onto the MEA substrate, and imaged the same day using phase contrast (top) and 2-photon fluorescence (bottom) microscopy. Four fields of view were imaged robotically for over 4 hours (two shown), with no effect on 
fluorescence or cell viability. The number ' 81 ' refers to the $8^{\text {th }}$ column and first row, the upper-right corner of the array of 60 electrodes. Images were collected every $10 \mathrm{~min}$. The time lapse sequence shows odd frames, at 20-min intervals, a zoom-in of the boxed region in the phase-contrast image above. Growth cones and numerous filipodia can be observed making contact with neighboring labeled and unlabeled cells.

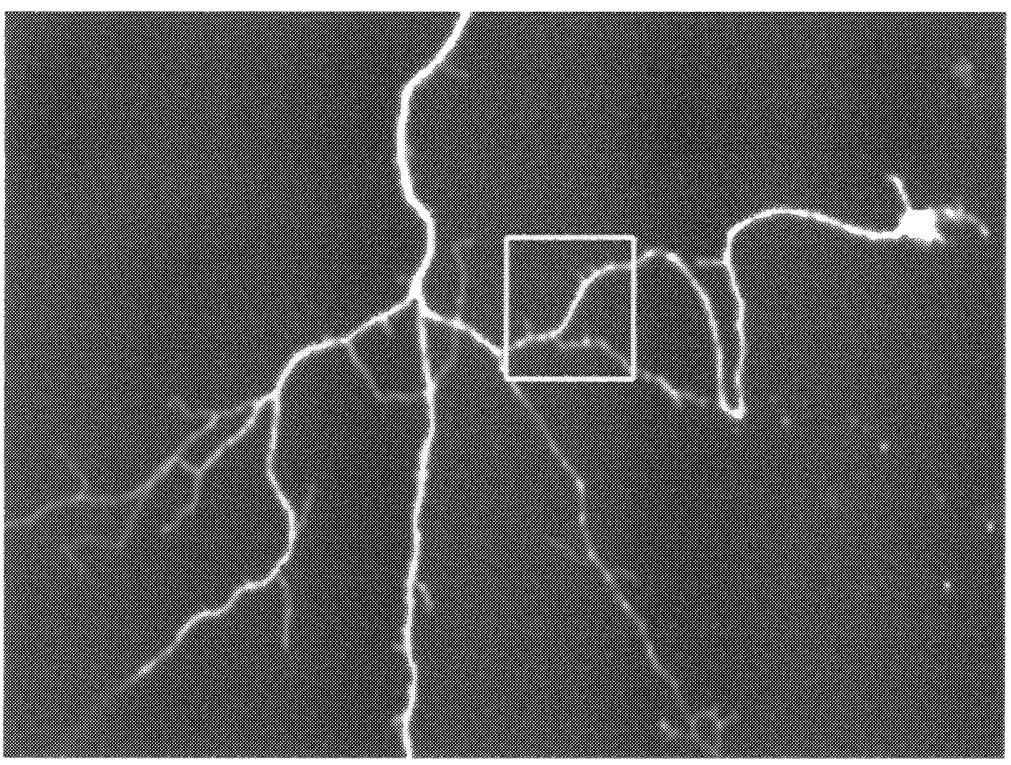

Fig. 6. Two-photon time-lapse movie of GFP liposome-labeled dendritic spines in a mature cortical culture. Rapid spontaneous synaptic remodeling is demonstrated in these frames, taken at 90 -sec intervals. A spine in frame 1 (arrow) is gone in the second frame, and one that appears in frame 4 (arrow) grows over the next $7 \mathrm{~min}$ to form a mature spine head with a thin stalk too narrow to resolve (frame 9, arrow). Zoomed region (white box, top image) is $16 \mu \mathrm{m}$ across.

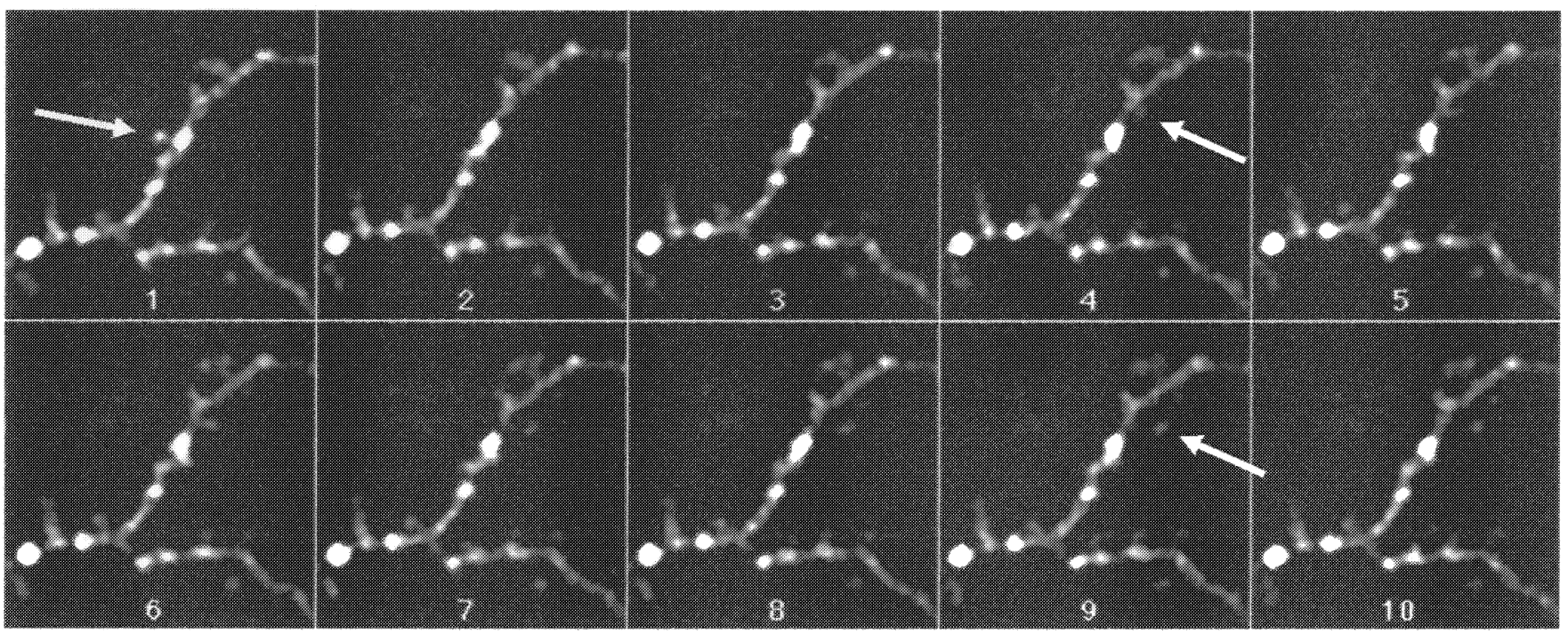

\section{CONCLUSION}

The brain is the most powerful computer we know of at this time. Perhaps if we understood more about how it processes and stores information, we could design much more powerful artificial computing systems, ones that utilize the massively parallel, distributed network dynamics that brains use. To help discover which morphological and electrical dynamics are important in neuronal networks, we have developed a number of new tools for studying many neurons in great detail at a variety of time scales. By connecting a cultured neuronal network to a computer on which the animal's body can be simulated, we have opened the door to the study of learning and memory in vitro. By imaging the neurons involved in the learning process while the learning is happening, and while recording their electrical activity via an array of electrodes, we have the potential toexamine links between electrical activity and morphology in a manner not possible with living animals. 


\section{ACKNOWLEDGEMENTS}

This work was funded in part by an RO1 grant from the National Institute of Neurological Disorders and Stroke, NS38628, and by the Beckman Foundation. We thank Profs. Scott E. Fraser and Jerome Pine for additional support, guidance, equipment, and lab space. We thank Tom DeMarse, Axel Blau, Daniel Wagenaar, Sam Thompson, Peter Meilstrup, Gray Rybka, Mike Atkin, Andrew Barajas, Sheri McKinney, and Sami Barghshoon for their efforts in the creation of the Neurally Controlled Animat. We thank Eric Murphy, Alan Waring, Sherry Haynes, Phil Felgner and Mary Dickinson for development and testing of the liposome-based gene delivery system.

\section{REFERENCES}

1. Weiler, I. J., Hawrylak, N. and Greenough, W. T., "Morphogenesis in Memory Formation - Synaptic and Cellular Mechanisms." Behavioural Brain Research 66: 1-6, 1995.

2. Pine, J., "Recording action potentials from cultured neurons with extracellular microcircuit electrodes." Journal of Neuroscience Methods 2: 19-31, 1980.

3. Gross, G. W., Rieske, E., Kreutzberg, G. W. and Meyer, A., "A new fixed-array multi-microelectrode system designed for long-term monitoring of extracellular single unit neuronal activity in vitro." Neuroscience Letters 6: 101-105, 1977.

4. Potter, S. M. and DeMarse, T. B., "A new approach to neural cell culture for studying long-term plasticity.":, (in preparation).

5. Banker, G. and Goslin, K. (1998). Culturing Nerve Cells, 2nd Edition. Cambridge, Mass., MIT Press.

6. Jimbo, Y., Tateno, T. and Robinson, H. P. C., "Simultaneous induction of pathway-specific potentiation and depression in networks of cortical neurons." Biophysical Journal 76: 670-678, 1999.

7. Robinson, H. P. C., Kawahara, M., Jimbo, Y., et al., "Periodic synchronized bursting and intracellular calcium transients elecited by low magnesium in cultured cortical neurons." Journal of Neurophysiology 70: 1606-1616, 1993.

8. Maeda, E., Robinson, H. P. C. and Kawana, A., "The mechanisms of generation and propagation of synchronized bursting in developing networks of cortical-neurons." Journal Of Neuroscience 15: 6834-6845, 1995.

9. Morefield, S. I., Keefer, E. W., Chapman, K. D. and Gross, G. W., "Drug evaluations using neuronal networks cultured on microelectrode arrays." Biosensors \& Bioelectronics 15: 383-396, 2000.

10. Kamioka, H., Maeda, E., Jimbo, Y., et al., "Spontaneous periodic synchronized bursting during formation of mature patterns of connections in cortical cultures." Neuroscience Letters 206: 109-112, 1996.

11. Tateno, T. and Jimbo, Y., "Activity-dependent enhancement in the reliability of correlated spike timings in cultured cortical neurons." Biological Cybernetics 80: 45-55, 1999.

12. Meyer, J.-A. and Guillot, A. (1994). From SAB90 to SAB94: Four years of animat research. From Animals to Animats 3: Proceedings of the Third International Conference on Simulation of Adaptive Behavior. D. Cliff, P. Husbands, J.-A. Meyer and S. W. Wilson. Cambridge, MIT Press: 2-11.

13. DeMarse, T. B., Wagenaar, D. A., Blau, A. W. and Potter, S. M., "Neurally-controlled computer-simulated animals: a new tool for studying learning and memory in vitro." Soc. Neruosci. Abstr. 26: 467.20, 2000.

14. Potter, S. M., Mart, A. N. and Pine, J., "High-speed CCD movie camera with random pixel selection, for neurobiology research." SPIE Proceedings 2869: 243-253, 1997.

15. Denk, W., Strickler, J. H. and Webb, W. W., "2-photon laser scanning fluorescence microscopy." Science 248: 73-76, 1990.

16. Potter, S. M., "Vital imaging: Two photons are better than one." Current Biology 6: 1595-1598, 1996.

17. Potter, S. M., Fraser, S. E. and Pine, J., "The greatly reduced photodamage of 2-photon microscopy enables extended 3dimensional time-lapse imaging of living neurons." Scanning 18: 147, 1996.

18. Potter, S. M. (2000). Two-Photon Microscopy for 4D Imaging of Living Neurons. Imaging Neurons: A Laboratory Manual. R. Yuste, F. Lanni and A. Konnerth. Cold Spring Harbor, CSHL Press: 20.1-20.16.

19. Potter, S. M., Wang, C. M., Garrity, P. A. and Fraser, S. E., "Intravital imaging of green fluorescent protein using 2photon laser-scanning microscopy." Gene 173: 25-31, 1996.

20. Potter, S. M., Pine, J. and Fraser, S. E., "Neural transplant staining with DiI and vital imaging by 2-photon laser-scanning microscopy." Scanning Microscopy Supplement 10: 189-199, 1996.

21. Longmuir, K. J., Murphy, E. A., Waring, A. J., Dickinson, M. E., and Haynes, S. M., "A nonviral liposomal complex designed to overcome the multiple barriers to gene transfer." Moleular Therapy 1: S243, 2000. 